\title{
On a Generalization of the Law of Sines to the Tetrahedron and Simplices of Higher Dimensions
}

\author{
M.A. Murray-Lasso \\ Unidad de Enseñanza Auxiliada por Computadora \\ Departamento de Ingeniería de Sistemas. División de Estudios de Posgrado \\ Facultad de Ingeniería, UNAM \\ E-mail: mamurray@ servidor.unam.mx
}

(recibido: enero de 2003; aceptado: mayo de 2003)

\begin{abstract}
Thewell known Law of Sines for tri an gles is carefully an a lyzed to gether with its stan dard proof and based on that anal $y$ sis it is ex tended to the tet ra hedron and simplices of four and moredimen sions. Thecru cial step in theproof of theex ten sion to thetet ra hedron starts by rep r esenting each tri an glein theskin (sur face) of the tet ra hedron as a vec tor in three-dimensional space whose mag ni tude is equal to thearea of the tri an gleand which is nor mal to the plane of thetrian gle. The sum of these four vec tors is thezero vector when thefaces are prop erly ori ented. The next step is to takethevectors and pro ject them u pon a di rected linethat issi mul ta neously perpen dicu lar to two of thevectors. The sum of theprojections must bezero, but becausethedirected lineis or thogo nal to two of them it must also beor thogo nal to thesum of thevectors rep rsent ing thetwo other faces of thetet ra hedron. Thereareat least two sim plegeomet rical in ter preta tions to themain result: first, choosing two dis joint pairs of faces of thetet ra hedron theedgejoin ing the first pair of faces is or thogo nal to the sum of the vec tors rep resent ing thetwo other faces; sec ond, thevol umes of two par al lel epi peds formed with trios of vector rep resen ta tion of facesof thetet ra hedron areequal. Theresult is ex tended to simplices of $n$ di men sions by rep resent ing the skin of the sim plex by $n$ vec tors or thogo nal to the hyperplanes where the el e ments of the skin lie. Since for $\mathrm{n}$-dimensional spaces it is pos si ble to find a vec tor vsimultaneaously or thogonal to $n-1$ vectors, the sameidea is ap plied and the pro jections of the sum of thelast two vectors rep re

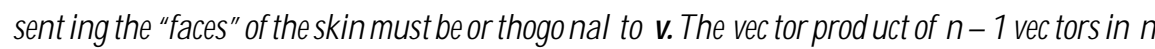
dimensional space is used to ob tain v. A sim plenu mer i cal ex am pleis given.
\end{abstract}

Keywords: Law of Sines, tet ra hedron, sim plex, pro jection, vector prod uct

\section{Resumen}

La conocida Ley de los Senos para los triángulos es analizada cuidadosamente junto con su demostración estándar, con base en dicho análisis se le extiende al tetraedro y a simplejos de cuatro y más dimensiones. El paso crucial en la demostración de la extensión al tetraedro comienza representando cada triángulo en la piel (superficie) del tetraedro como un vector en el espacio tridimensional, cuya magnitud es igual al área del triángulo y es normal al plano en el que está el triángulo. La suma de estos cuatro vectores es el vector cero cuando 
las caras están adecuadamente orientadas. El siguiente paso es tomar los vectores y proyectarlos sobre una línea dirigida, que es simultáneamente perpendic ular a dos de ellos. La suma de las proyecciones debe ser cero, pero debido a que la línea dirigida es ortogonal a dos de ellos, también debe ser ortogonal a la suma de los vecto res que representan a las dos caras restantes del tetraedro. Hay por lo menos dos interpretaciones geométricas sencillas del resultado principal; primero, si se escogen dos pares disjuntos de caras del tetraedro, la arista que los une al primer par es ortogonal a la suma de los vectores que representan las caras del segundo par; segundo, los volúmenes de dos paralelepípedos formados con dos trios de vectores que representan las caras son iguales. El resultado se extiende a simplejos de cuatro y más dimensiones, representando la piel del simplejo $n$-dimensional por medio de $n+1$ vectores ortogonales a los hiperplanos donde yacen los elementos de la piel. En vista de que en un espacio de $n$ dimensiones es posible encontrar un vector $\mathbf{v}$ simultáneamente ortogonal a $n-1$ vectores, se aplica la misma idea y las proyecciones de la suma de los últimos dos vectores que representan las "caras" de la piel del simplejo deben ser ortogonales a $\mathbf{v}$. Se utiliza el producto vectorial entre $n-1$ vectores en $n$ dimensiones para encontrar $\mathbf{v}$. Se muestra un ejemplo numérico.

Descriptores: Ley de los Senos, tetraedro, simplejo, proyección, producto vectorial.

\section{Introduction}

The Law of Sines is one of the important theorems of Plane Geometry and Trigonometry whose importance is at a par with the Law of Cosines and is right behind the Pythagorean The o rem, which ac cord ing to several au tho rs is the most important theorem in all of mathe matics (Davis and Hersh, 1980), (Wylie, 1964). In this paper we pres ent a genealization of the Law of Sines to the tetrahedron and to anal ogous ob jects in four and more di men sions. The tri an gle can be seen as a particularization of a tetrahedron that has zero height. It can also be seen as the con vex hull of 4 points in three dimensional space when two of the points are made to co in cide and the body flat tens into a two dimensional plane figure. In such a case the generalized Law of Sines for the tetrahe dron reduces to the Law of Sines for the triangle. In order to generalize the Law of Sines we use the vec tor prod uct of $n-1$ vec tors in $n-$ dimensional space (Murray-LAsso, 2004). It is to be noted that the extension presented in this paper to the Law of Sines is an equation relating areas of the faces for the case of the tetrahedron and hyperareas of the objects in the skin of the sim plex in the case of higher dimen sions. Altough many ex ten sions of the Law of Sines exist by applying the familiar Law of Sines to two-dimensional triangles that are formed or can be constructed in the tetrahe dron and higher dimensional simplices, these are not treated in this paper.

\section{The Proof of the Law of Sines for the Triangle}

In order to be able to generalize the Law of Sines to geometric ob jects whose di men sions 
exceeds two, it is necessary to carefully choose the proof of the original theorem and to view it in a mathematical environment as free as we can without destroying the final result.

The typical proof of the Law of Sines goes as follows (Ayres, 1954); (Gutiérrez-Ducons, 1985):

Con sider the trian gles shown in fig ure $1 a$ and $b$.

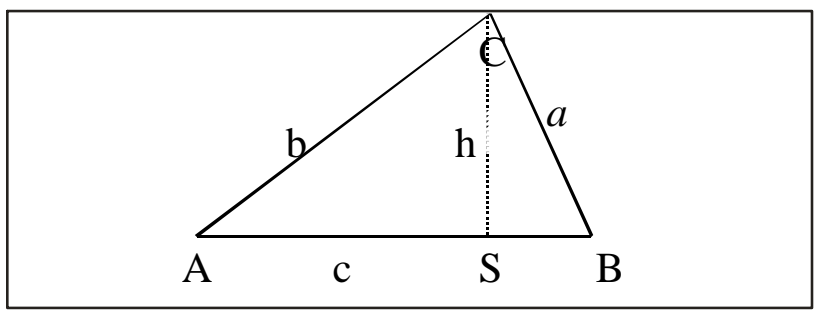

Figure 1 a

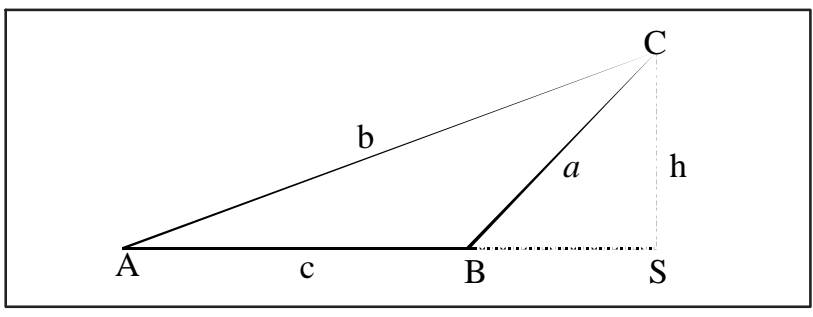

Figure 1b

The three vertices of the triangles are labelled $A, B$ and $C$ and we will use the same letters to denote the corresponding interior angles. The sides opposite to the verices are labelled with cor re spond ing lower case let ters a, $b$ and $c$. Fig ure la rep re sents the case in which the triangle is acuteangular while figure $1 b$ represents the case of an obtuseangular triangle. From ver tex $C$ we draw a line per pendic u lar to line $c$ - to its ex ten sion in case b) - so that we can cal cu late the length of line $h$ in two man ners:

$$
h=b \sin A=a \sin B
$$

the last mem ber for case b) be comes a sin ( 180 $-B)$, recalling that $B$ is the angle at vertex $B$ interior to the original triangle, but since sin $(180-B)=\sin B$, in both cases we obtain the same expression. From the second and third mem ber of equa tion (1) we ob tain

$$
\frac{b}{\sin B}=\frac{a}{\sin A}
$$

We now repeat the process changing the roles of the sides of the tri an gle, and from vertex $B$ we draw a perpendicular to line $b$ and reason in a similar fashion to obtain the expression

$$
\frac{c}{\sin C}=\frac{a}{\sin A}
$$

which togeteher with equation (2) can be written

$$
\frac{b}{\sin B}=\frac{a}{\sin A}=\frac{c}{\sin C}
$$

which is the Law of Sines.

For the purpose of generalizing the Law of Sines to more dimensions it is convenient to consider the triangle as the geometric object associated with the vec tor sum $a+b+c$ of three vec tors (ar rows) in a space with 2 or more di men sions using the tri an glelaw or poly gon law and its corresponding algebraic expressions for vec tor ad di tion, that is, in the case of geometricalinterpretation the tail of a vector coin cides with the arrow of the pre vi ous vec tor in the sum as shown in figure 2. (In figure 2 we have drawn things as though $A, B$ and $C$ are in the same plane, which must be the case if the space is two-dimensional. See figure 5 for the case where they are not). 


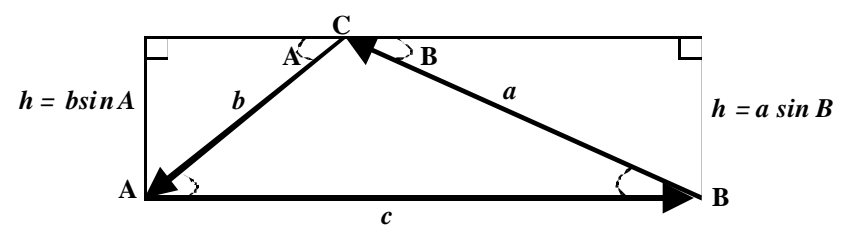

Figure 2

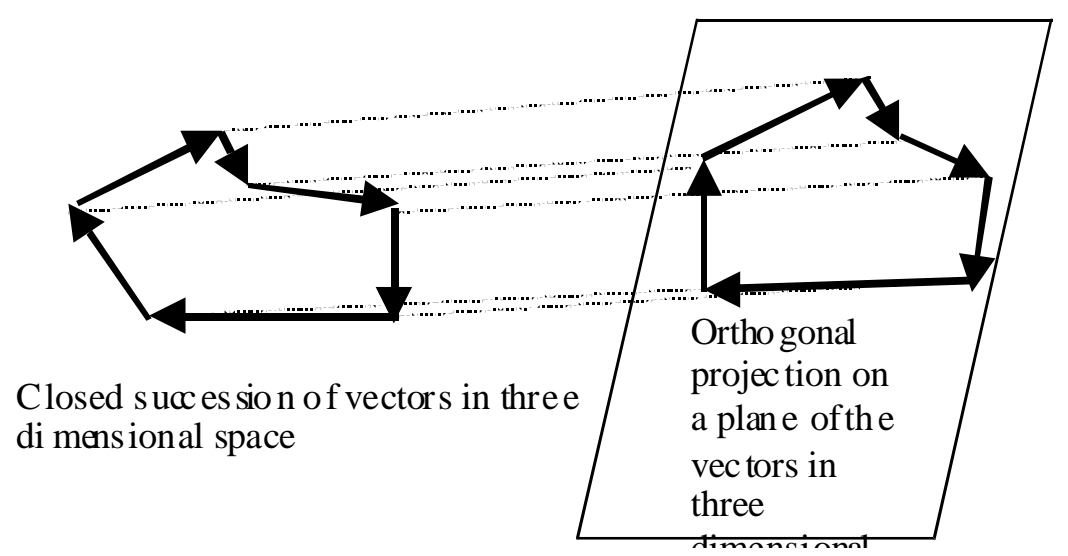

Figure 3

Since in the case of a triangle, the polygon is closed, the final sum will be the zero vector. Now, when we have a figure constructed from ar rows suc ceed ing each other and fi nally closing on itself, not only is the vector sum the zero vec tor, but also the vec tor sum of the orthogonal projections of these vectors upon any subspace of lower di men sion. Fig ure 3 illus trates the idea. In the proof of the Sine Law the space for pro ject ing is a line or thogo nal to one of the sides, say the hor i zon tal side. In this case the horizontal line has zero orthogonal projection upon the line orthogonal to it and only two sides have a non zero projection. Since the two projections must add to zero, the magnitudes of the proyections must be equal.

The projections of vectors $\mathbf{a}$ and $\mathbf{b}$ upon $\mathbf{a}$ line or thogo nal to $\mathrm{c}$ do not have to be thought of as passing through point $\mathrm{C}$, the important point is that they have the same magnitude; their val ues are $h=|\mathbf{b}| \sin A=|\mathbf{a}| \sin B$, (the an gles marked $A$ and $B$ are equal to the in terior angles at the vertices $A$ and $B$ because they are al ter nate in te rior an gles be tween paral lel lines) from which the Law of Sines fol lows by re peat ing the argu ment for lines or thogo nal to ei ther side $\mathbf{b}$ or side $\mathbf{a}$. It is this in terpretation that will allow us to see a gen er al iza tion of the Law of Sines to higher dimensional objects.

\section{The Law of Sines for the Tetrahedron}

To generalize the Law of Sines to the tetrahedron we rep re sent each of its faces with a vector whose magnitude is the absolute value of the area of the face and whose direction is ortogonal to the plane of the face (Spiegel, 1998). To de cide on the di rec tion of the vec tor we orient the faces by defining a sense in 
which the rim of the face is tra versed (this can bedone by ordering the vertices in a partic ular way) and as so ciat ing the ar rows of the vec tors with the "right hand screw rule," which says that the arrow points in the direction in which a right hand screw would advance when it is turned in the sense in which the rim of the surface is tra versed (Spiegel, 1998). This is shown in figure 4

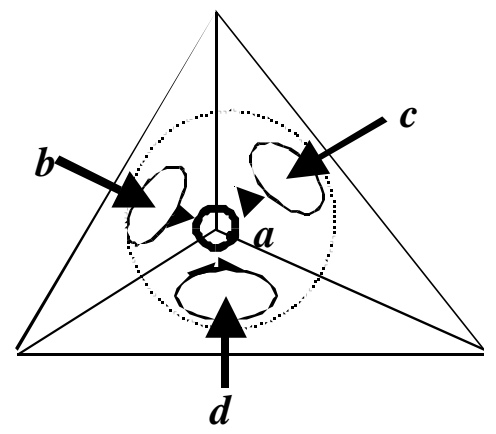

Figure 4

In figure 4 we are ob serving a tet ra he dron as seen from above. The orien ta tions of the faces are so cho sen that all ar rows enter the tet ra hedron. The arrow cor re spond ing to the hor izontal plane is drawn as a circle with a dot in the middle to represent an arrow pointing directly to wards the ob server. The dot ted di rected circle is the di rec tion of tra versal of the horizontal face. The vec tor sum of the four ar rows representing the faces in three dimensionalspace have a zero sum, therefore, when arranged in space ac cord ing to the poly gon law, they form a four-edge three-dimensional polygon that closes upon itself (Spiegel, 1998). As mentioned above, the projection of this closed arrow poly gon upon any subspace must be the zero vec tor in the said subspace. To look for a sine law we choose a subspace for projecting consisting of a line such that only two of the vec tors rep re sent ing the faces of the tet ra he dron have non zero projections upon the line.
Since we are in a three dimensional space, if we form the vector product of two of the vectors rep re sent ing the faces of the tet ra he dron, the re sult ing vec tor will be or thogo nal to both vector factors and both will have zero projections along the line. The pro jec tions on the line of the other two face-representing vectors (which can be ob tained through a dot prod uct) must be equal in mag ni tude. Let us choose the face-representing vectors $\mathbf{a}$ and $\mathbf{b}$ as the vectors that will have zero projections, then equating the magnitudes of the projections of the other two vectors we obtain the following expression

$$
|\mathbf{a} \times \mathbf{b} \cdot \mathbf{c}|=|\mathbf{a} \times \mathbf{b} \cdot \mathbf{d}| *
$$

where we have cancel led a factor $|\mathbf{a} \times \mathbf{b}|$ dividing both members of equation (4). By choosing other pairs of vectors for the cross prod uct and re peat ing the ar gu ment we ob tain (the meaning of the asterisk's is explained below)

$$
\begin{aligned}
&|\mathbf{a} \times \mathbf{c} \cdot \mathbf{b}|=|\mathbf{a} \times \mathbf{c} \cdot \mathbf{d}| * \\
&|\mathbf{a} \times \mathbf{d} \cdot \mathbf{c}|=\mathbf{a} \times \mathbf{d} \cdot \mathbf{b} \\
& \mathbf{c} \times \mathbf{b} \cdot \mathbf{a}=\mid \mathbf{c} \times \mathbf{b} \cdot \mathbf{d} *
\end{aligned}
$$

$$
|\mathbf{b} \times \mathbf{c} \cdot \mathbf{d}=| \mathbf{b} \times \mathbf{c} \cdot \mathbf{a} \mid
$$

and several others. The equations are not all in de pend ent due to the prop erties of the tri ple product. Using the brackett notation (Hsu, 1986) equa tions (7) and (8) can be writ ten

$$
\begin{aligned}
& |c, b, a=| c, b, d \mid \\
& |b, c, d=| b, c, a \mid
\end{aligned}
$$


Taking into account that a cyclic permutation of the letters in the bracketts does not change its value and that an ordinary permutation changes the sign (Hsu, 1986) the last equation can be writ ten

$$
-[c, b, d]=-[c, b, a]
$$

Cancelling both negative signs in this last equa tion re veals that equa tion (8) is re ally the same equation as (7). Only the equations marked with an as ter isk are in de pend ent in the set of equa tions (4) to (8). The three equa tions with asterisk (or other equivalent ones) are a vec tor form of the Law of Sines for the tet ra hedron. Notice that it is an equation relating areas of faces, not lengths of lines.

\section{Geometric Interpretations}

Equation (4) can be given the following interpretation:

The cross product $\mathbf{a} \times \mathbf{b}$ produces a vector that is orthogonal to both $\mathbf{a}$ and $\mathbf{b}$. Being orthogonal to vectora implies it lies on a plane paral lel to (that is with the same ori en ta tion) as face $A$ of the tet ra hedron. Being or thogo nal to vec torb im plies it lies in a plane with the same orientation as face $B$ of the tetrahedron. Imposing both conditions simultaneously means it lies in a line whose ori en ta tion is the same as the intersection of faces $A$ and $B$, that is, the edge that joins faces $A$ and $B$. The vector can be normalized to unit length by dividing it by the scalar $|\mathbf{a} \times \mathbf{b}|$ (although that will not be nec es sary because this fac tor can be can cel led with the same factor appearing on the right member.) The dot product (of the normalized vector) with vector $\mathbf{c}$ gives sim ply the pro jection of $\mathbf{c}$ upon the edge men tioned. This takes care of the left side of equation (4). Similarily the right hand side represents the projection of the vector $\mathbf{d}$ upon the same edge (as sum ing we still have not taken out the nor mal iz ing factor $|\mathbf{a} \times \mathbf{b}|$ ). After cancel lingthenormal izing factor on both sides of equation (4) what we have is the magnitudes of both projections mul tiplied by the factor $|\mathbf{a} \times \mathbf{b}|$. Thein terpreta tion can be ap plied to to other pairs of vectors representing the areas of the faces of the tetrahedrons. We note that to obtain any of the formu las we choose the vec tors as so ciated with a a pair of faces; their cross prod uct determines a vector in the direction of the edge be tween the faces. The mag ni tudes of the dot products of the two vectors associated with the re main ing two faces are then equal to each other.

Since the algebraic sum of the projections of the four vectors representing the faces of a tetrahedron upon any di rected line is zero and when the line chosen is orthogonal to two of them, say $\mathbf{a}$ and $\mathbf{b}$, then the sum of the pro jections of vectors $\mathbf{c}$ and $\mathbf{d}$ gives the zero vec tor. This means that the fol low ing equa tion holds

$$
(\mathbf{a} \times \mathbf{b}) \cdot(\mathbf{c}+\mathbf{d})=\mathbf{0}
$$

in other words, the cross prod uct of any two of the vectors representing two faces are orthogonal to the sum of the vectors rep resent ing the two re main ing faces.

A sec ond in ter pre ta tion can be given in terms of vol umes, since a triple prod uct can be as so ciated with the volume of a parallelepiped whose sides meeting at a vertex are the vectors in the tri ple prod uct. The Law of Sines can then be inter preted as the equal ity be tween the vol umes of the parallelepipeds defined by vectors $\mathbf{a}, \mathbf{b}, \mathbf{c}$ and $\mathbf{a}, \mathbf{b}, \mathbf{d}$. Several ad ditional equal ities between vol umes can be ob tained by permutting the vectors. Re call that the vec tors $\mathbf{a}, \mathbf{b}, \mathbf{c}, \mathbf{d}$ are vec tors 
orthogonal to the faces of the tetrahedron of mag ni tudes equal to their areas.

The reader may won der whether we can obtain for the tetrahedron expres sions similarto the equa tions ap pear ing in the Law of Sines for tri an gles. We cer tainly can, since for any closed four sided polygon of three-dimensional vectors closing upon themselves (that is, such that the sum of the vec tors is the zero vec tor) the algebraic sum of the projections of the vec tors with re spect to a di rected line or thogonal to two of them (which for non zero vectors can always be obtained through the cross product) is zero, we have a situation such as that de picted in fig ure 5 .

The pro jec tions of $\mathbf{a}$ and $\mathbf{b}$ upon $\mathbf{z}$ are given by

$\operatorname{Proj}_{\mathbf{z}} \mathbf{a}=\mathbf{a}, \mathbf{z} /|\mathbf{z}|=\mathbf{n}|\mathbf{a}| \cos \theta=\mathbf{n}|\mathbf{a}| \sin \beta$

$\operatorname{Proj}_{\mathbf{z}} \mathbf{b}=\mathbf{b} \cdot \mathbf{z} /|\mathbf{z}|=\mathbf{n}|\mathbf{b}| \cos \varphi=\mathbf{n}|\mathbf{b}| \sin \alpha$

Where $\mathbf{n}$ is a unit vec tor in the di rec tion of $\mathbf{z}$. Since both pro jections are equal in magni tude we have $|\mathbf{b}||\sin \alpha|=|\mathbf{a}||\sin \beta|$

from which a part of the Law of Sines is obtained in the form

$$
\frac{\mathbf{a} \mid}{\sin \alpha \mid}=\frac{|\mathbf{b}|}{|\sin \beta|}
$$

with the geometric interpretation that the angles are between vectors representing two faces of the tetrahedron and perpendicular lines in the same plane as a line which is si multaneously orthogonal to the two other faces and the vector such as a representing a face. By applying the same argument to additional pairs of vectors representing faces we can deduce a se ries of expres sions of the form

$$
\frac{a}{\sin \alpha \mid}=\frac{b}{|\sin \beta|} ; \frac{c}{|\sin \gamma|}=\frac{d}{\sin \delta} ; \frac{e}{\sin \varepsilon \mid}=\frac{f}{|\sin \kappa|}, \ldots
$$

where the letters $a, b, c, d, e, f$ represent magnitudes of vec tors and the an gles have sim i lar geometric interpretations. It is necessary to in troduce different angles for different pairs of faces
These two segments are gener ally not collinear
The three vec tors $\boldsymbol{a}, \boldsymbol{b}, \boldsymbol{z}$, meet in a common point $\mathrm{O}$

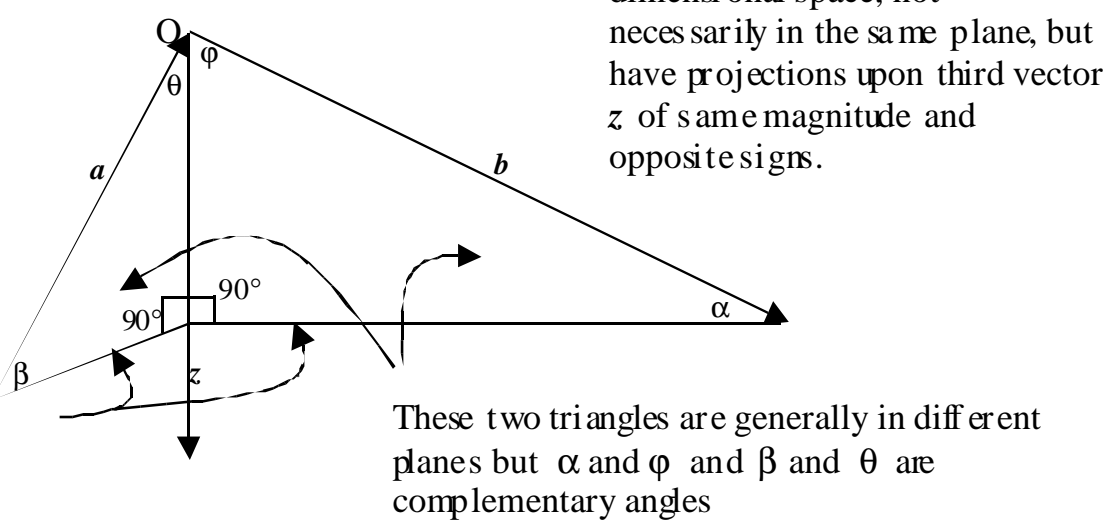

Third vector $z$ in the direction of

$\boldsymbol{c} \times \boldsymbol{d}$, and the ref ore orthogonal to $\boldsymbol{c}$ and $\boldsymbol{d}$ ( $\boldsymbol{c}$ and $\boldsymbol{d}$ are not shown)

Figure 5 
becausetheircross prod uct determines different vectors play ing the role of $\mathbf{z}$ in figure 5 .

\section{Numerical Illustrative Example}

Con sider a tet ra he dron such as the one shown in figure 6 , in which the coordinates with respect to $C$ ar te sian axes $x, y, z$ are given for the verti ces $A, B, C, D$.

In figure 6 the vertices of the tetrahedron are labelled $A, B, C, D$ and close to them are trios of num ber in pa ren the ses with their co ofdinates. The vectors representing the faces of the tet ra hedron arela belled $a, b, c, d$ while the faces themselves are labelled $A^{\prime}, B^{\prime}, C^{\prime}, D^{\prime}$. Each of the edges has been given a sense. The sense of traversal of each of the faces is such that the vectors representing them are all directed leaving the tetrahedron. The vectors representing the edges are as fol lows:

Edge DC: $(0.5,1,0)-(0,0,0)=(0.5,1,0)$

Edge $D B:(1,0,0)-(0,0,0)=(1,0,0)$

Edge $A B:(1,0,0)-(0.2,0,1)=(0.8,0,-1)$

Edge $A C:(0.5,1,0)-(0.2,0,1)=(0.3,1,-1)$

Edge DA : $(0.2,0,1)-(0,0,0)=(0.2,0,1)$
The vectorrep resentation $v$ of a face by a vector normal to it and whose magnitude is given by the area of the face, in the case of trian gles is given by

$$
v=1 / 2\left(v_{1} \times v_{2}\right)
$$

where $\mathbf{v}_{1}$ and $\mathbf{v}_{2}$ are two adjacent edges of the triangles and, given a sense of traversal of the rim of the triangle, the order of the factors in the product is chosen so that when the first vector is rotated through an angle less than 180 de grees, so as to make it co in cide in di rection and sense with the second vector, the turn ing is in the sense of tra versal of the rim of the area rep re sented. Using these con cepts we find:

$$
\begin{aligned}
& \mathbf{a}=1 / 2(B C \times D C)=(0,0,-0.5) \\
& \mathbf{b}=1 / 2(D A \times D C)=(-0.5,0.25,0.1) \\
& \mathbf{c}=1 / 2(D B \times D A)=(0,-0.5,0) \\
& \mathbf{d}=1 / 2(A B \times A C)=(0.5,0.25,0.4)
\end{aligned}
$$

No tice that the sum of the four vec tors representing the faces is the zero vector.

We now pro ceed to check whether equa tion (4) is sat is fied.

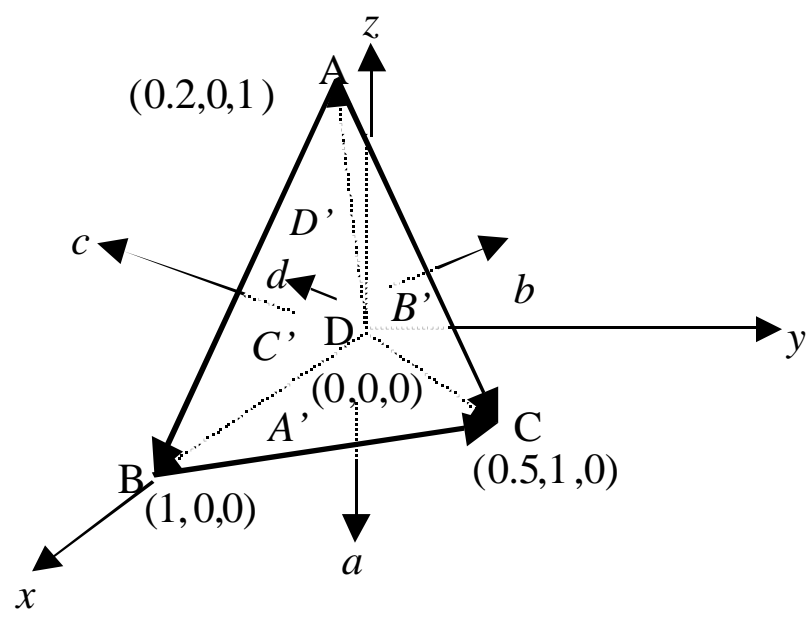

Figure 6 

$\mathbf{a} \times \mathbf{b}=(0.125,0.25,0)$
$\mathbf{a} \times \mathbf{b} \cdot \mathbf{c}=-0.125 \quad \mathbf{a} \times \mathbf{b} \cdot \mathbf{d}=0.125$

Since the two magnitudes are equal, equation (4) is satisfied. The orthognality property can be checked in a second way. The vector $D C$ be tween faces $\mathbf{a}$ and $\mathbf{b}$ is

$$
(0.5,1,0)
$$

This vector must be orthogonal to the sum c+d.

$$
\begin{gathered}
\mathbf{c}+\mathbf{d}=(0,-0.5,0)+(0.5,0.25,0.4)= \\
(0.5,-0.25,0.4)
\end{gathered}
$$

The orthogonality between the two vectors can be checked via the dot prod uct

$$
\begin{gathered}
(0.5,1,0) \cdot(0.5,-0.25,0.4)= \\
0.25-0.25=0
\end{gathered}
$$

Since the dot product is zero, the two vectors are orthogonal. In a similar fashion we ob tain

$$
|\mathbf{c} \times \mathbf{b} \cdot \mathbf{a}|=|\mathbf{c} \times \mathbf{b} \cdot \mathbf{d}|=0.125
$$

there fore equa tion (7) is sat is fied. By choos ing dif fer ent pairs of vec tors the Law can be tested for the other cases. We leave the verifications to the reader.

\section{Extension to More Dimensions}

The extension of the Law of Sines to simplices of more dimensions is straight for ward. A simplex in four dimensions, for instance, is a geo met ric ob ject that has a three $\mathrm{di}$ mensional skin consisting of 5 three-dimensional tetrahedrons (which is the number of possible combinations of 5 points taken 4 at a time), in analogy to a tetrahedron in three dimen sions that has a two-dimensional skin consisting of 4 ( number of combinations of 4 points taken 3 at a time) two-dimensional tri angles. Each one of the tetrahedrons of the four-dimensional simplex is a piece of a 3-dimensional hyperplane and can be repre sented in four-dimensional space through a four-dimensional vector whose direction is orthogonal to the hyperplane and whose magnitude is equal to the vol ume of the corre sponding tetrahedron. Now in a four-dimensional space we can find a vector which is simulta neously orthogonal to three four-dimensional vectors (a generalization to one more dimension of the fact that in a two dimensional space we can find a vec tor or thogo nal to onevec tor; in a three dimensional space we can find a vector that is simultaneously orthogonal to two vectors.) One way to find them is solving a set of homogeneous linear equations with a zero determinant. A second way is through the cross prod uct of three vec tors in 4-dimensions. A convenient definition for this product in terms of the cartesian components of the four-dimensional vec tors $\mathbf{a}, \mathbf{b}$, and $\mathbf{c}$ is

$$
\mathbf{a} \times \mathbf{b} \times \mathbf{c}=\left|\begin{array}{cccc}
a_{1} & a_{2} & a_{3} & a_{4} \\
b_{1} & b_{2} & b_{3} & b_{4} \\
c_{1} & c_{2} & c_{2} & c_{4} \\
\mathbf{i} & \mathbf{j} & \mathbf{k} & \mathbf{I}
\end{array}\right|
$$

where $\mathbf{i}, \mathbf{j}, \mathbf{k}$, I are unit vec to rs in the di rec tion of an orthogonal right-handed system of axes. The re sult of the cross prod uct of the three vectors is a four dimensional vector, that it is orthogonal to all three vectors can be eas ily seen by per form ing the dot prod uct with each one of them in succession. The 
mixed prod uct $(\mathbf{a} \times \mathbf{b} \times \mathbf{c})$. $\mathbf{d}$ is a sca lar that can be writ ten

$$
\left(\mathbf{i} p_{1}+\mathbf{j} p_{2}+\mathbf{k} p_{3}+I p_{4}\right) \cdot\left(\mathbf{i} d_{1}+\mathbf{j} d_{2}+\mathbf{k}\right.
$$
$d_{3}+\mid\left(d_{4}\right)=p_{1} d_{1}+p_{2} d_{2}+p_{3} d_{3}+p_{4} d_{4}$

where $p_{1}, p_{2}, p_{3}, p_{4}$ are the components of the product $\mathbf{a} \times \mathbf{b} \times \mathbf{c}$ and $d_{1}, d_{2}, d_{3}, d_{4}$ are the com po nents of $\boldsymbol{d}$. This last sca lar can be written as the following determinant

$$
\left|\begin{array}{llll}
a_{1} & a_{2} & a_{3} & a_{4} \\
b_{1} & b_{2} & b_{3} & b_{4} \\
c_{1} & c_{2} & c_{3} & c_{4} \\
d_{1} & d_{2} & d_{3} & d_{4}
\end{array}\right|
$$

If $\mathbf{d}$ is equal to any of $\mathbf{a}, \mathbf{b}, \mathbf{c}$ the de terminant will have two iden ti cal rows, and there fore ac cord ing to a well known the o rem of de ter mi nants (Thomas, 1960) the value of the de ter $\mathrm{mi}$ nant will be zero, establishing the orthogonality of the three-vector cross prod uct with each of its fac tors.

Once we know how to find a vector orthogonal to three vectors in four dimensional space, given the five four-dimensional vectors representing the tet ra he dra that forms the skin of the four-dimensional simplex, vectors that when we properly orient the tetrahedra add to the zero vec tor, we find a vec tor or tho go nal to three of them and with it and the two remaining vectors representing two of the tetrahedra we form two triangles in four dimensions for which figure 5 applies and hence we can extend the Law of Sines to this case. The ex pression of the Law can be written

$$
(\mathbf{a} \times \mathbf{b} \times \mathbf{c}) \cdot(\mathbf{d}+\mathbf{e})=0
$$

where $\mathbf{a}, \mathbf{b}, \mathbf{c}, \mathbf{d}$, e are four-dimensional vectors representing the tetrahedra of the skin of the four-dimensional sim plex. Many sim i lar expressions can be obtained by interchanging the vec tors.

Generalizing to $n$ dimensions, for a simplex in $n$ di men sions whose skin is formed by $n+1$ simplices of $n-1$ di men sions rep re sented by $n+1$ vectors orthogonal to their hyperplanes, the expressions associated with the Law of Sines take the form

$$
\left(\mathbf{v}_{1} \times \mathbf{v}_{2} \times \ldots \times \mathbf{v}_{n-1}\right) \cdot\left(\mathbf{v}_{\mathrm{n}}+\mathbf{v}_{\mathrm{n}+1}\right)=0
$$

where $\mathbf{v}_{1}, \mathbf{v}_{2}, \ldots, \mathbf{v}_{n+1}$ are the vectorrep resentations of the ( $n-1)$-dimensional simplices con form ing the skin of the n-dimensional simplex. Additional expressions may be obtained by per mut ing the in di ces of the vec tors.

The last equation is also valid for a two-dimensional sim plex, that is for a tri an gle, if the vector product is properly interpreted. The vec to rexpres sion for the trian gle reads

$$
\mathbf{a} \times \cdot(\mathbf{b}+\mathbf{c})=0
$$

In this case the vec tor prod uct has only one factor and its calculation can be done using the determinantal expression

$$
\begin{array}{cc}
a_{1} & a_{2} \\
\mathbf{i} & \mathbf{j}
\end{array} \mid=-\mathbf{i}_{2}+\mathbf{j} \mathbf{a}_{1}
$$

which is orthogonal to the vector $\mathbf{i} a_{1}+\mathbf{j} a_{2}$ which plays the role of the horizontal side of the triangle in the original proof of the Sine Law. It is for rea sons such as this that it is more con ve nient to con sider the vec tor prod uct in $n$ di men sions as a func tion of $n-1$ or dered variables than as a bi nary op era tion and there fore thenotation $\times(\mathbf{a} ; \mathbf{b} ; \mathbf{c})$ is to be pre ferred to $\mathbf{a}$ $\times \mathbf{b} \times \mathbf{c}$.

The sides of the tri an gle rep re sented by orthogo nal vec tors is shown in fig ure 7. 


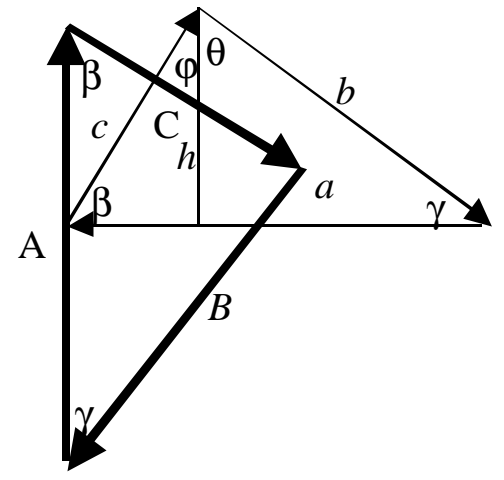

Figure 7

In figure 7 the original triangle is shown in heavy lines, its sides are labelled with upper case let ters. The vec tors or thogo nal to the sides of the original triangle, rotated positively through 90 and of magnitudes equal to the lengths of the orig i nal sides are la belled with the corresponding lower case let ters. The two tri angles are congruent, one is simply a rotation of the other, hence $A=a, B=b, C=c$ and the angles la belled with the same let ters are equal. No tice also that both tri an gles are tra versed in a clockwise direction when the traversal coincides with the senses of the arrows. Since the arrows add to the zero vector the conditions stated for the proof of the Law of Sines hold. The hor i zon tal side of the tri an gle in thin lines a is chosen as the side that will have a zero projection upon a vertical line (orthogonal to the horizontal side a). The pro jec tions of the sides $b$ and cupon the ver ti cal line $h$ are

$b \cos \theta=b \sin \gamma=c \cos \varphi=c \sin \beta$

from which

$$
\frac{c}{\sin \gamma}=\frac{b}{\sin \beta}=\frac{C}{\sin \gamma}=\frac{B}{\sin \beta}
$$

and the rest of the pro of fol lows by now tak ing a second side as the one having zero projection upon a line or thogo nal to the side.

\section{Conclusions}

We have ex tended the fa mil iar Law of Sines of the triangle to the tetrahedron and to simplices of four and more dimensions. The law re lates the areas and hyperareas of the el $\mathrm{e}$ ments of the skin of the body. Hence for the tetrahedron it relates areas of triangular faces, for the 4-dimensional simplex it relates volumes of tet ra he dra form ing the skin of the simplex, etc. To do it con ve niently we es tab lished a vector expression that is equivalent to the Law of Sines. The proof was accomplished by taking a slightly different view of the ingredi ents in the stan dard proof of the Law of Sines for the tri an gle. The vec to r prod uct of $n-1$ vectors in $n$-dimensional space came in very handy for obtaining a vector that is simulta neously orthogonal to all the vector factors which is an essential part of the proof of the Law of Sines.

\section{References}

Ayres Jr.F. (1954). Plane and Spherical Trigonometry. Schaum Publishing Company, New York.

Davis P.J. and Hersh R. (1980). The M athematical Experience. Birkhäuser, Boston.

Gutiérrez-Ducons J.L. (1985). M atemáticas, geometría y trigonometría. Gran Enciclopedia Científica Cultural. Organización Cultural LP, SA de CV, México.

Hsu H.P. (1986). Análisis vecto rial. Wilmington, DE. Addi son-Wesley Iberoamericana.

Murray-Lasso M.A. (2204). Sobre el producto cruz en espacios vectoriales $\mathrm{N}$-dimensionales, Ingeniería Investigación y Tecnología, Vol.V, No.1, pp.27-46.

Spiegel - Murray R. (1998). A nálisis vecto rial. Mc Graw-Hill, México. 
Thomas S.B. (1960). Calculus and Analytic

Geometry. Reading, MA. Addison-Wesley

Publishing Company.

Wylie Jr. C.R. (1964). Foun da tions of G eom etry.

Mc Graw-Hill Book Company, New York.

\section{Semblanza del autor}

M arco Antonio M urray-Lasso. Realizó la licenciatura en ingeniería mecánica-eléctrica en la Facultad de Ingeniería de la UNAM. El Instituto de Tecnología de Massachussetts (MIT) le otorgó los grados de maestro en ciencias en ingeniería eléctrica y doctor en ciencias cibernéticas. En México, ha laborado como investigador en el Instituto de Ingeniería y como profesor en la Facultad de Ingeniería (UNAM) durante 43 años; en el extranjero, ha sido asesor de la NASA en diseño de circuitos por computadora para aplicaciones espaciales, investigadoren los Laboratorios Bell, así como profesor de la Universidad Case Western Reserve y Newark College of Engineering, en los Estados Unidos. Fue el presidente fundador de la Academia Nacional de Ingeniería de México; vicepresidente y pres i dente del Consejo de Academias de Ingeniería y Ciencias Tecnológicas (Organización Mundial con sede en Washington que agrupa las Academias Nacionales de Ingeniería) y secretario de Ia Academia Mexicana de Ciencias. Actualmente es jefe de la Unidad de Enseñanza Auxiliada por Computadora de la División de Estudios de Posgrado de la Facultad de Ingeniería de la UNAM, investigador nacional en ingeniería, consejero educativo del MIT y consultor de la UNESCO. 